\title{
Artículos
}

\section{Conceptos de web 2.0 y biblioteca 2.0: origen, definiciones y retos para las bibliotecas actuales}

\author{
Por Dídac Margaix Arnal
}

Resumen: Se expone una visión global de los conceptos web 2.0 y biblioteca 2.0. En primer lugar se analizan varios sitios web considerados de éxito y que dieron lugar a la aparición de este concepto, posteriormente se analizan las características esenciales de este tipo de webs desarrollando las principales novedades tecnológicas y de relación con el usuario. En la segunda parte del trabajo se analiza el concepto de biblioteca 2.0 como aplicación de la web 2.0. Tras un breve repaso a las principales definiciones se propone una nueva definición. Se añaden ejemplos de los servicios 2.0 que pueden ofrecer o están ofreciendo las bibliotecas.

Palabras clave: Web 2.0, Biblioteca 2.0, Software social, Inteligencia colectiva, Servicios bibliotecarios, Definiciones.

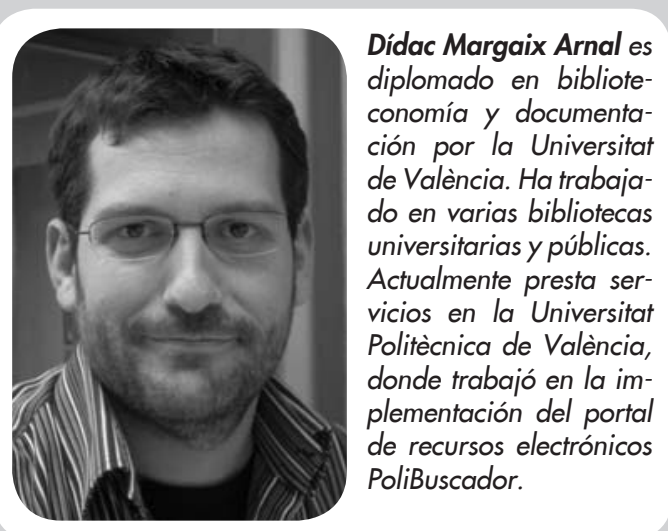

Title: Concepts of web 2.0 and Library 2.0: origin, definitions and challenges for today's libraries

Abstract: This article provides an overview of web 2.0 and library 2.0. An analysis is offered of websites widely acknowledged as being successful. These websites were those that led to the new concept web 2.0. Essential characteristics of this type of site are reviewed, such as employing important technologic innovations and novel ways of interacting with users. The second part of the paper analyses the significance of library 2.0 as a web 2.0 application for libraries. After a brief review of the principal definitions, a new one is proposed. Examples are given of the 2.0 services that libraries are currently offering or could offer in the future.

Keywords: Web 2.0, Library 2.0, Social software, Collective intelligence, Library services, Definitions.

Margaix Arnal, Dídac. "Conceptos de web 2.0 y biblioteca 2.0: origen, definiciones y retos para las bibliotecas actuales". En: El profesional de la información, 2007, marzo-abril, v. 16, n. 2, pp. 95-106.

DOI: 10.3145/epi.2007.mar.01

\section{Introducción}

Una nueva revolución está agitando el entorno de internet, se llama web 2.0. Este concepto está empezando a generar nueva literatura profesional tratando de describir este planteamiento y aplicarlo a distintos ámbitos, como el periodismo, la educación o las bibliotecas. Pero ésta no es la primera ni la última revolución que viven las bibliotecas y se ha de tener especial cuidado en no repetir frases hechas que ya han sido utilizadas en otras ocasiones y fijarnos en los aspectos novedosos que puede aportar la web 2.0 al trabajo de los profesionales de la información.

En este artículo se pretende dar una visión global de las implicaciones que conlleva así como su aplicación al contexto bibliotecario, la biblioteca 2.0. Para ello se realiza un breve análisis de los sitios web que mejor representan esta filosofía. A continuación se explica el origen del concepto y sus implicaciones, haciendo hincapié en los aspectos tecnológicos y en los de relación con el usuario. Precisamente son los nuevos modelos de vínculo con el usuario donde la web 2.0 ha tenido un mayor calado y más implicaciones en el desarrollo de nuevos servicios. El último apartado del artículo está dedicado al concepto de biblioteca 2.0, explicando su origen y tratando de clarificarlo, dando unas claves que permitan identificar y establecer nuevos servicios en bibliotecas y revisando algunos ejemplos. 


\section{El concepto web 2.0}

En otoño de 2001 se produjo el estallido de la burbuja tecnológica, lo que provocó que muchas empresas desaparecieran del mercado o vieran fuertemente reducidas sus expectativas, pero eso no pasó con todas. Algunas sobrevivieron y otras aparecieron con nuevas ideas y nuevos modelos de negocio. En el año 2004 dos empresas del sector informático, O'Reilly y MediaLive International, realizaron un brainstorming para analizar aquellas que habían superado la crisis y poder identificar las características claves de éxito. A las que no lo superaron las denominaron "web 1.0" y a las que sí, o estaban teniendo éxito en ese momento las llamaron "web 2.0" (O'Reilly, 2005a).

Esto nos da idea de las primeras características del concepto. En primer lugar podemos decir que nace de la observación y no de un replanteamiento teórico de los servicios de internet. Había servicios web 2.0 antes de formularse el concepto. En segundo lugar, nace en entorno empresarial y tiene, en su origen, una marcada impronta tecnológica y de marketing. La tercera característica es que no hay un elemento clave que sitúe un servicio web en el lado del 2.0, existe una serie de características que se pueden cumplir en mayor o menor medida, pero es difícil encontrarlas todas simultáneamente en un mismo servicio web.

En su primer trabajo O'Reilly (2005a) no da una definición de web 2.0, así que cuando se le pidió esa definición envió un mensaje al blog de su empresa (O'Reilly, 2005b) definiéndola. Afirmó que las aplicaciones web 2.0 son aquellas que sacan partido a las ventajas intrínsecas de la web, ofreciendo un servicio continuamente actualizado que mejora cuanto más gente lo use, utilizando y remezclando los datos de múltiples recursos, incluyendo los usuarios individuales, a la vez que ofrecen sus propios datos y servicios de tal forma que pueden ser reutilizados por otros, creando una "arquitectura de participación" en red, yendo más allá de la página de la web 1.0 para ofrecer experiencias de usuario cada vez más ricas.

En esta definición puede verse claramente cómo el autor está relacionando la web 2.0 con la tecnología casi de forma exclusiva. Pero son los principios de compartir, reutilizar, mejora continua, consideración del usuario como fuente de información, confianza,

"No hay que repetir frases hechas que ya han sido utilizadas en otras ocasiones y fijarnos en los aspectos novedosos que puede aportar la web 2.0" aprovechamiento de la inteligencia colectiva, etc., los que han impulsado el establecimiento de la actitud 2.0, haciendo que la tecnología pase a un segundo plano.

En la actualidad cuando se habla de web 2.0 se está haciendo referencia al uso de determinadas tecnologías (ajax, mashups, software social, rss) y a la aplicación de una determinada actitud en el diseño de servicios web, basada en los principios citados.

\section{Ejemplos de servicios web 2.0}

Existen muchos más, pero con estos siete pueden verse reflejados todos los principios.

- Amazon. Esta librería virtual constituye uno de los modelos de éxito más conocidos y consolidados de internet. Comenzó a operar en julio de 1995 y en aquel momento contaba con la misma información sobre sus productos que el resto de competidores, pero aprovechó la tecnología para dar a sus usuarios una experiencia de compra personalizada, recopilando la opinión de los propios lectores sobre los productos y aprovechando los datos de los clientes anteriores para ofrecer una información más adecuada y pertinente a los nuevos visitantes. Desarrolló una "ciencia de la gestión del usuario" (O’Reilly, 2005a; Bausch, 2003).

- Wikipedia es una enciclopedia libre, en donde los usuarios pueden consultar gratuitamente todos sus artículos, pero también permite libremente crear, ampliar y modificar entradas. Existen versiones activas en 171 lenguas y la edición inglesa cuenta con casi millón y medio de artículos. Aunque es un proyecto arriesgado, y no está exento de críticas y problemas, varios autores han demostrado su utilidad y fiabilidad (Austin, 2005; Gilles, 2005; Field, 2006).

- Del.icio.us fue pionero en lo que se denomina "social bookmarking" o marcadores sociales. Estos servicios permiten a los usuarios registrados guardar sus "bookmarks" (marcadores o favoritos) en el servidor web y asignar libremente unas palabras clave llamadas etiquetas o "tags" para describir y recuperar el enlace. Permite buscar por etiquetas y ver cuántos usuarios han seleccionado esa página web como favorita (GordonMurnane, 2006; Rethlefsen, 2006).

- Google Maps es un sistema de búsqueda de localizaciones geográficas. Las claves de su éxito son dos: en primer lugar utilizar las tecnologías ajax (asynchronous javascript and $\mathrm{xml}$ ) y en segundo lugar crear una API (application programming interface) que permite a los desarrolladores externos crear aplicaciones nuevas utilizando gratuitamente la información de Google Maps. Actualmente está considerada una de las herramientas web más completas y potentes (Lerner, 2006). 


\section{Sitios importantes web $\mathbf{2 . 0}$}

- Amazon.

http://www.amazon.com

- Del.icio.us.

http://del.icio.us

- eBay. El web de subastas por excelencia ofrece toda una serie de sistemas de valoraciones de compradores y vendedores. Sus contenidos son alimentados fundamentalmente por los propios usuarios.

http://www.ebay.es

- Flickr. Permite compartir fotografías y ofrece múltiples sis- temas de vinculación entre los distintos usuarios.

http://www.flickr.com

- GMail.

http://gmail.google.com

- Google Maps.

http://maps.google.com

- HousingMaps. Combina una base de datos inmobiliaria con Google Maps para mostrar la localización de los inmuebles que cumplen los resultados de la búsqueda. Es el ejemplo de mashup más citado. http://www.housingmaps. com

- Panoramio. http://www.panoramio.com

- Wikipedia. http://www.wikipedia.org

- YouTube. http://www.youtube.com

- Best of the best web 2.0 web sites. Selección de los mejores sitios web 2.0.

http://www.realsoftwaredevelopment.com/2006/10/ best_of_the_bes.html
- Flickr aprovechó el auge de las cámaras digitales y permite a sus usuarios compartir, comentar y guardar sus fotografías. Tiene servicios gratuitos y de pago, pero prohíbe la venta de fotografías. Nació en 2004 y fueron los usuarios de blogs los que le dieron un importante impulso al usarlo para publicar sus fotografías. En marzo de 2005 Yahoo! compró la empresa (Sinclair, 2006).

- YouTube permite compartir vídeos. Cualquier persona registrada puede subirlos y añadir un comen- tario y unas etiquetas para describirlo, pero el resto de usuarios pueden darle una puntuación (acción conocida como rating), añadir comentarios, seleccionarlo como favorito, etc.

- Panoramio. Puede ser el ejemplo español de sitio web 2.0. Consiste en un servicio para compartir fotografías, similar a Flickr, pero a través de un sencillo sistema y utilizando las imágenes de Google Maps es posible indicar la ubicación desde donde se tomó la fotografía así como buscar todas aquellas tomadas en un

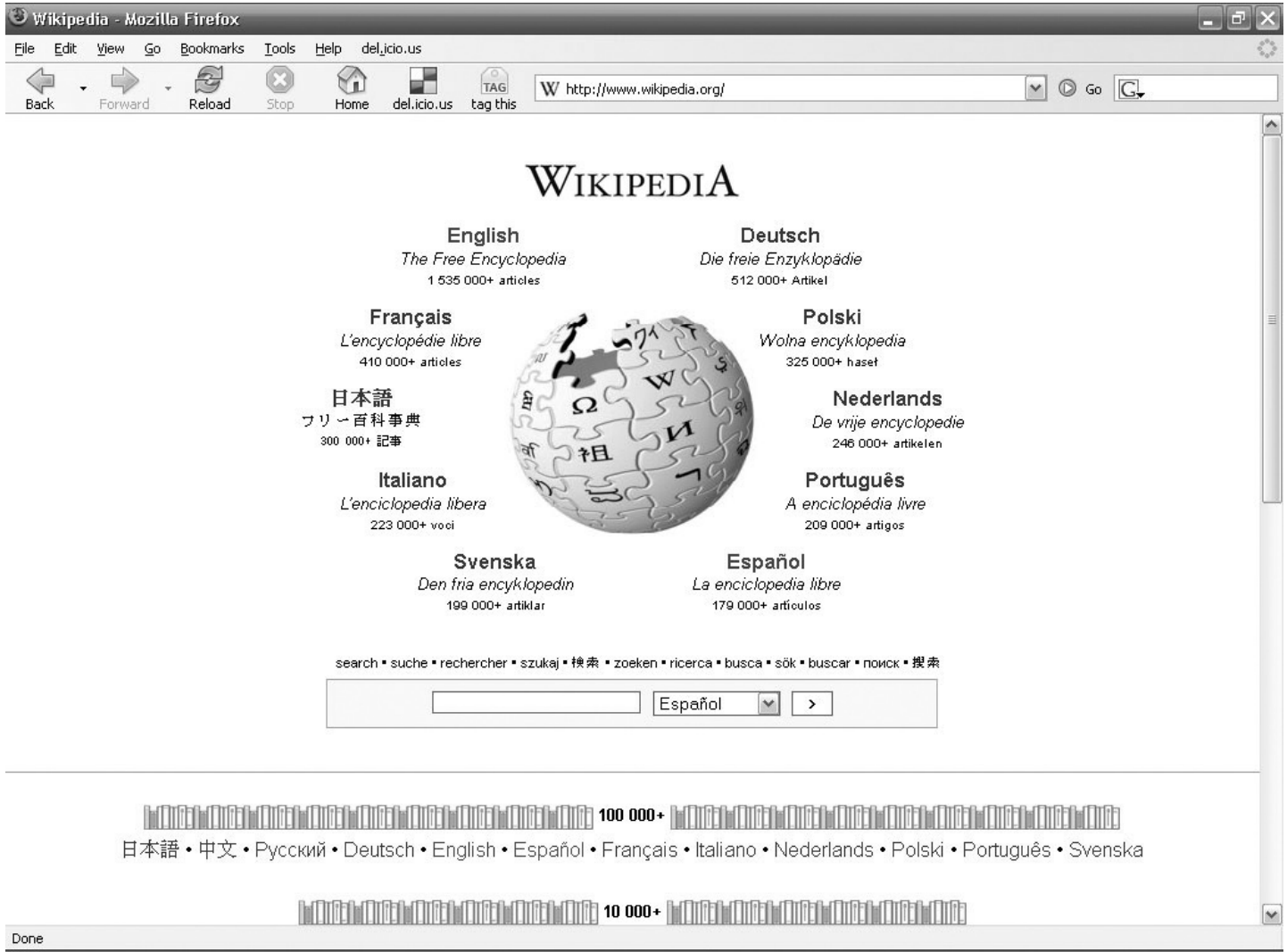

Página principal de Wikipedia 


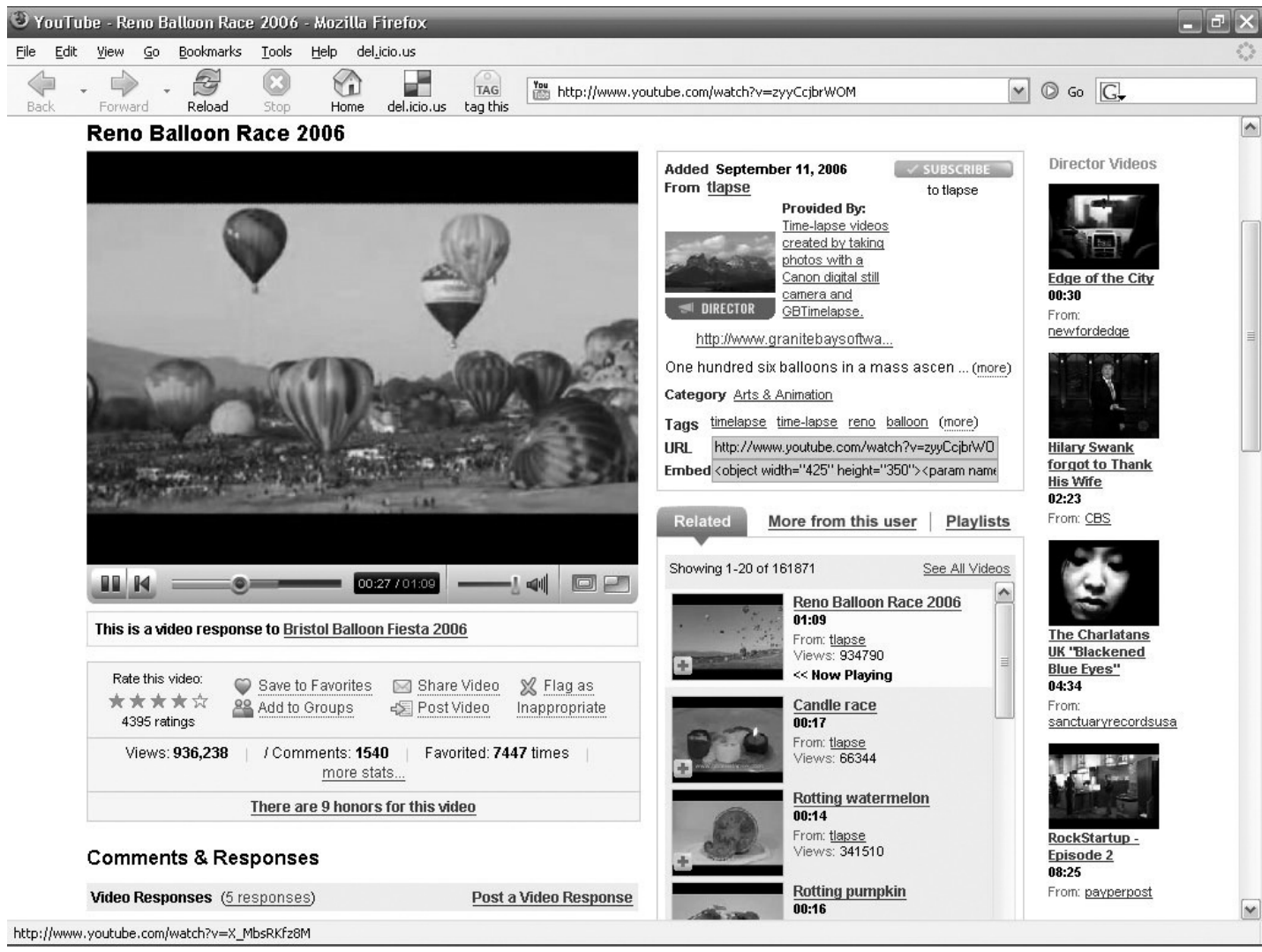

YouTube contiene muchos elementos de aprovechamiento de la inteligencia colectiva

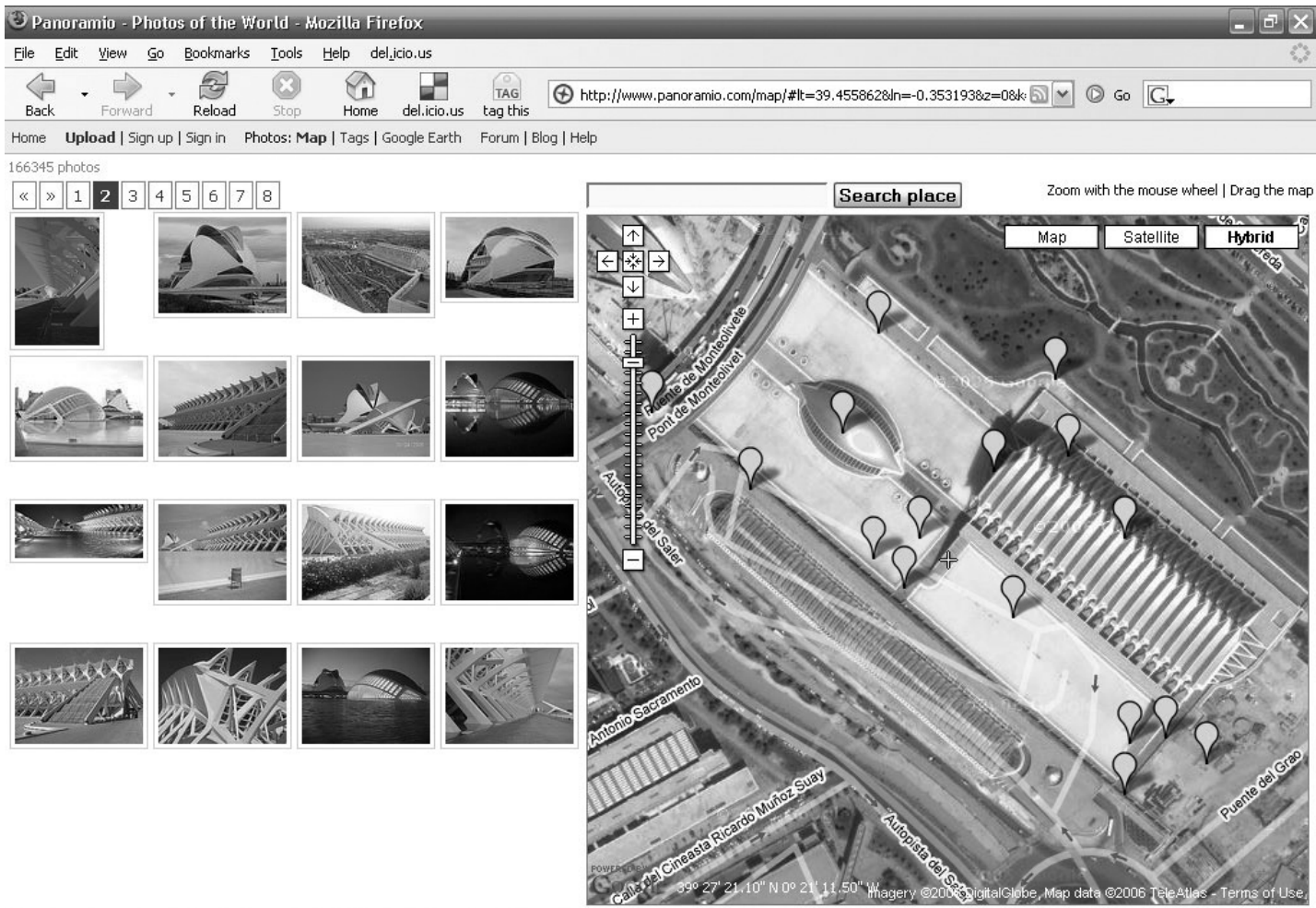

Copyright @ 2005, 2006 Ainda.info. All rights reserved. | Photos are under the copyright of their owners. | Blog | About | Contact us

http://www. panoramio.com/map/\#

Panoramio es un ejemplo de mashup, remezclando los datos de Google Maps con las fotos de los usuarios 
área geográfica concreta. Actualmente es posible visualizar las fotografías de Panoramio mientras se navega por Google Earth.

\section{Las tecnologías 2.0}

Hay algunas tecnologías consideradas 2.0 , es decir, suelen estar presentes en los sitios web 2.0. No siempre son originarias de la web 2.0, pero son consideradas típicas de estos sitios:

- Ajax: una combinación de xml y javascript que posibilita crear aplicaciones web ejecutables en el cliente, reduciendo considerablemente el tráfico de datos y la carga de trabajo del servidor, y con una mayor interactividad (Lerner, 2006). Muchos de los casos de "experiencias de usuario ricas" están programados con este lenguaje, como puede ser Google Maps, GMail o Flickr.

- Los mashups o aplicaciones web híbridas. Integran de manera transparente los datos de otros servicios web, gracias a que algunos de éstos han creado APIs que permiten desarrollar nuevas aplicaciones que accedan a sus datos gratuitamente. El ejemplo más citado es HousingMaps.com, donde se combina la información de una base de datos inmobiliaria con Google Maps.

- El software social supone un elemento clave en todo el desarrollo de la web 2.0, especialmente en lo

\section{"La auténtica revolución 2.0 es la nueva concepción que se tiene de los usuarios, de manera que interactúen entre ellos y con el personal de la biblioteca"}

que se refiere al aprovechamiento de la inteligencia colectiva. Se entiende como tal el conjunto de aplicaciones que permiten a los individuos comunicarse unos con otros y seguir las conversaciones a través de la web (Tepper, 2003). Actualmente se suelen incluir también aquellas herramientas y servicios que permiten compartir información y objetos digitales, como los vídeos de YouTube o las fotografías de Flickr. Dentro de esta categoría hay tres componentes que han influido de manera decisiva en el la web 2.0: los blogs, los foros y las wikis.

a. Los blogs, también llamados weblogs o bitácoras, son páginas web que contienen mensajes con información ordenados cronológicamente y enviados por uno o varios autores. Normalmente permiten al resto de internautas leerlos y escribir comentarios. Actualmente es muy frecuente que los periódicos tengan los suyos propios, elaborados por periodistas en plantilla, desarrollando una nueva forma de columnas de opinión con

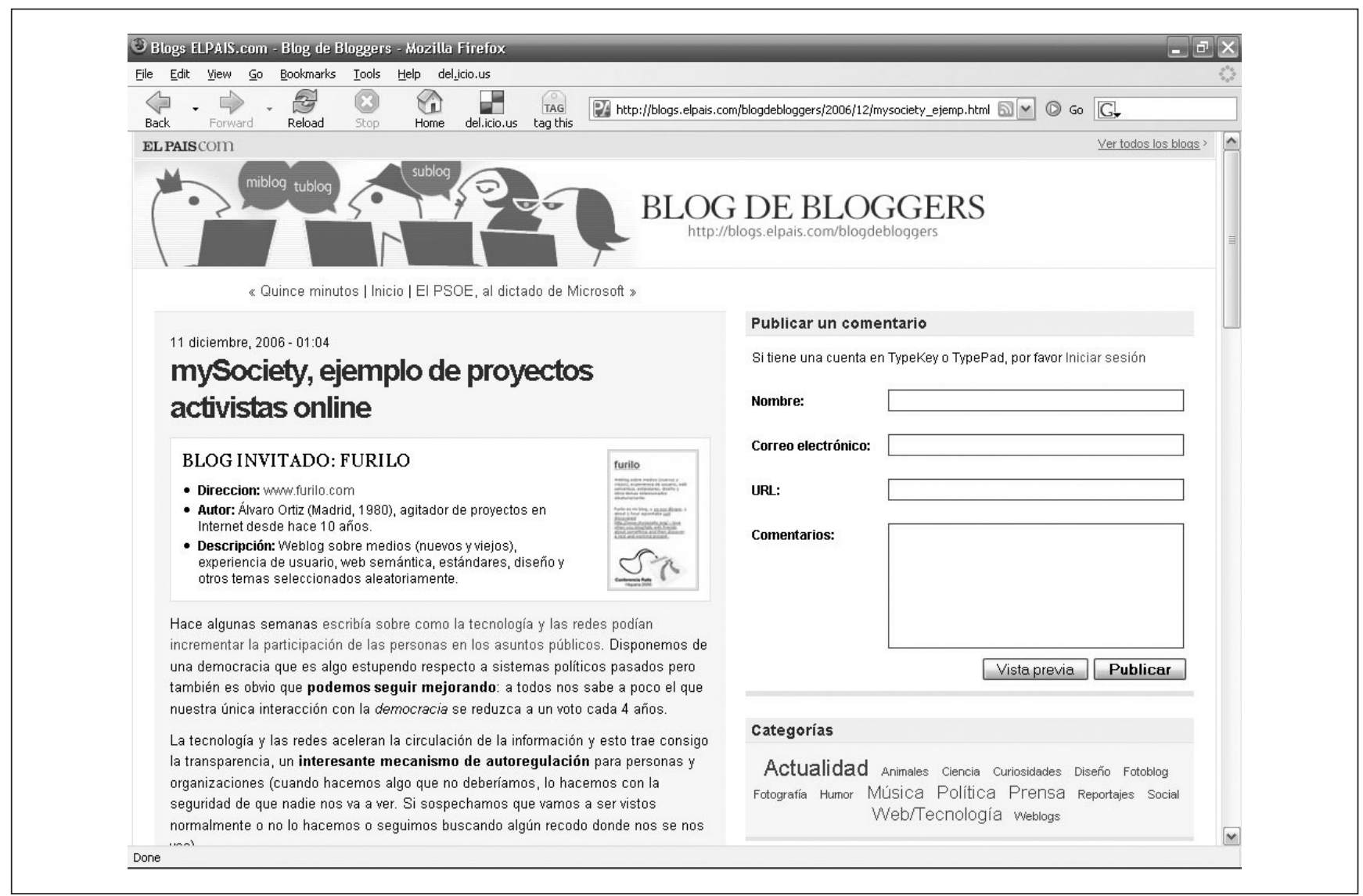

Blog de Bloggers en la página de Elpais.com 


\section{"El reto consiste en diseñar servicios atractivos, que sean útiles, e inviten a participar y a aportar conocimiento"}

un estilo más fresco y adecuado a la comunicación por internet. Véanse los ejemplos de Elpais.com, Elmundo. es o 20minutos.es. El efecto y sus posibilidades en las bibliotecas ha sido ya objeto de estudio de varios autores (Clyde, 2004; Franganillo, 2005). También existen muchos blogs dedicados a la biblioteconomía y la documentación, sirvan de ejemplo Catorze blog, El documentalista enredado y Deakialli documental.

b. Los foros tienen una forma similar de trabajar a los blogs. Permiten a cualquier usuario registrado plantear un tema de discusión y el resto puede participar en ella. Los mensajes se organizan en "hilos" que permiten seguir las conversaciones. Existen varios ejemplos de foros, pero los de la Asociación de Internautas pueden ser citados como ejemplo. Los foros tuvieron sus predecesores en la web 1.0: fueron los grupos de noticias o usenet y las listas de correo (p. ej., IweTel). Ambos servicios surgieron casi en los orígenes de internet y suponen los primeros sistemas para compartir información entre grupos de usuarios.

c. Las wikis son el modelo de software que permite a cualquiera leer, escribir y modificar mensajes escritos por cualquier otro usuario. El caso más famoso es Wikipedia, pero su software, Mediawiki, es utilizado para establecer wikis como sistema de comunicación entre grupos de intereses específicos o de trabajo. Un ejemplo en el entorno bibliotecario puede ser DigiRep Wiki, desarrollada en el marco del JISC Digital Repositories Program. También se puede citar la página de GUDE (Grupo de Usuarios Dspace de España), que han hecho de la wiki la web que gestiona todos sus contenidos.

- El rss es una familia de formatos xml para difundir información y ser reutilizada por otros programas o sitios web. Este proceso se denomina agregación o sindicación. Fue popularizado por los blogs y actualmente se encuentra implantado en webs de instituciones, en la mayoría de portales de revistas, bases de datos y en algunos catálogos de bibliotecas (Wusteman, 2004).

\section{Perspectiva hacia los usuarios en la web}

\section{0}

La auténtica revolución viene de la concepción que se tiene de los usuarios: se ha pasado de hablar de ellos como consumidores de información a tratarlos en términos de carácter participativo en la elaboración y gestión de los contenidos. Este cambio es debido al desarrollo de los principios de confianza radical y aprovechamiento de la inteligencia colectiva.

La confianza radical en el usuario implica fiarse plenamente en ellos, en sus acciones, en el uso que harán de los servicios, etc. Es la idea que subyace en proyectos como Wikipedia. Si no hay confianza total no tiene ningún sentido abrir espacios para la participación. No existe ninguna base para creer que la información de Wikipedia, un post de un blog o un comentario sobre un libro en Amazon son malintencionados o equivocados $\mathrm{y}$, aunque lo fueran, existen mecanismos de control.

El aprovechamiento de la inteligencia colectiva suele realizarse mediante la utilización del software social e integra todas las actividades encaminadas a recoger y utilizar el conocimiento de los propios usuarios de un servicio web. No basta con ser capaces de recopilar la información, ésta ha de ser utilizable por los siguientes usuarios, por ejemplo, el comentario de un libro en Amazon sólo es útil si otros clientes lo pueden leer. Este aprovechamiento se puede realizar de las siguientes formas:

- Crear contenidos por parte de los usuarios, como en el caso de los blogs y las wikis. Los usuarios registran su conocimiento, crean nueva información.

- Compartir objetos digitales, como pueden ser vídeos, fotografías, documentos o enlaces favoritos.

- Aportar comentarios a los objetos digitales compartidos. Por ejemplo, sobre los vídeos de YouTube, un libro en Amazon, o una opinión sobre el contenido del mensaje de un blog.

\section{Ejemplos de blogs, foros y wikis}

- Blogs de El país. http://www.elpais.com/blogs/

- Blogs de El mundo. http://www.elmundo.es/elmundo/blogs.html

- Blogs de 20Minutos. http://www.20minutos.es/blogs_opinion/
- Foros de la Asociación de Internautas. http://foros.internautas.org/

- DigiRep Wiki. http://www.ukoln.ac.uk/repositories/digirep/

- Página web de GUDE. http://sod.upc.es/gude/ 
- Incluir etiquetas (tagging) que describan el contenido de un objeto digital.

- Realizar valoraciones mediante puntuaciones (rating).

- Otras informaciones que se generan por el propio uso del servicio, básicamente a partir de los análisis de los logs de los servidores.

- Funcionalidades concretas de algunos sitios web, por ejemplo, el número de usuarios que han seleccionado un enlace como favorito en del.icio.us o los títulos que han comprado los usuarios en Amazon.

Todas estas formas de aprovechar el conocimiento colectivo tienen una característica común: el servicio mejora cuantos más usuarios tenga, es decir, cuantos más comentarios sobre libros proporcione Amazon, más útil será este servicio para los propios usuarios.

La confianza radical y el aprovechamiento de la inteligencia colectiva no son cambios tecnológicos, son cambios de actitud y, junto con otros elementos, han supuesto la base de lo que se llama la actitud 2.0 (O'Reilly, 2005a; Serrano, 2006). Dentro de esta filosofía se crean servicios orientados a la participación, no al uso. Los usuarios aportan un valor esencial al servicio y la información es compartida. El nuevo reto va a consistir en diseñar servicios atractivos, que sean útiles para los usuarios y les invite a participar y a aportar su conocimiento.

\section{Biblioteca 2.0}

Hasta ahora se ha dado una visión general sobre la web 2.0 y se ha descrito la condición sobre la que está

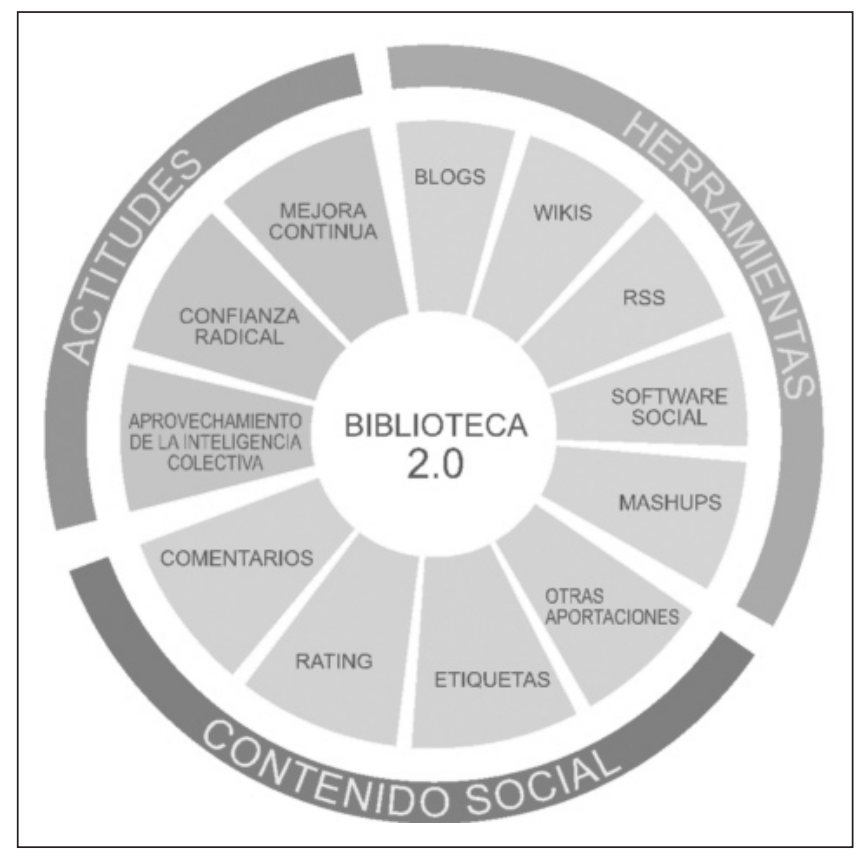

Elementos básicos de la biblioteca 2.0
"El concepto web 2.0 tiene una vertiente tecnológica y otra de relación con el usuario"

construida, la cual ha sido aplicada a diversos ámbitos, como el periodismo o la docencia, y se habla de periodismo 2.0 o de profesor 2.0 (Peña, 2006) y también se aplica al contexto de las bibliotecas.

\section{Origen del término biblioteca 2.0}

Según afirman Miller (2006) y Crawford (2006) fue acuñado en octubre 2005 por Michael Casey en su blog LibraryCrunch y estableció su primera definición en Wikipedia. El blog de Casey, junto al de Michael Stephens (Tame the web) y al de ALA TechSource, son las tres fuentes de información más importantes para observar cómo se está desarrollando el concepto.

Una importante característica es que el nacimiento del término y su debate conceptual se dan en la biblioblogosfera, es decir en el conjunto de blogs relacionados con la biblioteconomía (Ros, 2006). Esto produce que exista cierto volumen de información, pero muy dispersa en textos relativamente breves y que se puedan encontrar ciertas contradicciones entre unos blogs y otros (Crawford, 2006). También hay que indicar que la mayoría de los pioneros de la biblioteca 2.0 desempeñan su trabajo en bibliotecas públicas y son éstas las que están aplicando más rápidamente sus planteamientos y elaborando el concepto.

\section{En busca de una definición}

Al igual que sucede con otros planteamientos novedosos existen múltiples definiciones. Casey, que ha matizado su definición en diversas ocasiones, defiende una postura donde el usuario, el cambio constante y la evaluación continua son el centro de la biblioteca 2.0 (Casey, 2006). Algunos autores (Chad, 2005) ponen el énfasis en el uso de las tecnologías propias de la web 2.0. En esta misma línea se sitúa Maness (2006), que la conceptualiza como la aplicación de tecnologías interactivas, participativas y multimedia a los servicios y colecciones bibliotecarios basados en web. Paul Miller (2005) opta por una sencilla ecuación: "web $2.0+$ biblioteca = biblioteca 2.0". Michael C. Habib (2006a), plantea en un primer momento que es la aplicación y la adaptación del modelo web 2.0 al entorno bibliotecario, tanto virtual como físico y posteriormente (Habib, 2006b), en un trabajo mucho más exhaustivo, la define como los servicios bibliotecarios diseñados para cubrir las necesidades de los usuarios causadas directa o indirectamente por los efectos de la web 2.0. 


\section{Blogs importantes sobre biblioteca 2.0}

- Library Crunch. Blog de Michael Casey donde se pueden seguir sus comentarios y propuestas. http://www.librarycrunch.com/

- Tame the web. El blog de Michael Stephens es interesante por las novedades tecnológicas que presenta y por la forma de elaborar el blog.

http://www.tametheweb.com/

- ALA TechSource. Contiene información sobre novedades tecnológicas.

http://www.techsource.ala.org/blog/

- LIS. Blog personal de Michael Habib, donde expone avances sobre bibliotecas universitarias 2.0. http://mchabib.com/

\section{Blogs españoles sobre bibliotecas}

- Catorze blog.

http://www.catorze.com/blog/

- El documentalista enredado.

http://www. documentalistaenredado.net/

- Deakialli DocuMental.

http://www. deakialli.com/

- DosPuntoCero.

http://dospuntocero.dmaweb.info

\section{Ejemplos de servicios 2.0 en las biblio- tecas}

- Links-Yale Medical Library. http://links.med.yale.edu/

- PennTags. Descripción del proyecto de "social bookmarking" de la Universidad de Pennsylvania. http://tags.library.upenn.edu/help

- Ann Arbor District Library. http://www.aadl.org

- Biblioteca Pública de Muskiz. http://muskiz.com/b2

- Biblioteca Virtual Miguel de Cervantes. http://www.cervantesvirtual.com/

- WPopac. http://www.plymouth.edu/library/opac/

- Penn Library Toolbars. Cualquier usuario puede instalarse esta barra de herramientas en su navegador Firefox. http://labs.library.upenn.edu/toolbars/toolbars. html

- LibraryThing. Permite a los usuarios registrados catalogar sus propios libros y compartir información. Obtiene los datos de varios catálogos de bibliotecas usando el protocolo Z39.50, de Amazon y de sus propios usuarios. Es importante observar cómo presenta la información, podría ser el modelo del opac 2.0, también llamado "opac social". http://www.librarything.com/
Como puede observarse, la disparidad entre las definiciones es importante, pero de ellas y de los muchos mensajes en blogs (véase la recopilación de Crawford, 2006) se pueden extraer ciertos planteamientos generalizados:

- La biblioteca 2.0 deriva de la web 2.0.

- La tecnología es importante, pero no lo es todo.

- El usuario ha de tener un nuevo papel en la elaboración y gestión de los contenidos, se han de crear espacios para su participación.

- Biblioteca 2.0 hace referencia a los servicios y a las colecciones.

- Biblioteca 2.0 está en relación con el entorno virtual, pero también con el físico.

- Para ser un bibliotecario 2.0 se ha de perder el miedo a las tecnologías y a innovar.

Con todo esto llega el momento de proponer una nueva definición y, en una línea próxima a Habib, definir la biblioteca 2.0 como la aplicación de las tecnologías y la filosofía de la web 2.0 a las colecciones y los servicios bibliotecarios, tanto en un entorno virtual como real.

\section{Servicios 2.0}

Imaginemos una biblioteca pública de un municipio cualquiera. Ha conseguido una serie de fotografías antiguas y decide digitalizarlas y publicarlas en internet. Esta publicación puede hacerse a través de una serie de páginas html o mediante su catálogo bibliográfico. Estas opciones, correctas y legítimas, serían consideradas web 1.0. Ahora bien, supongamos que se decide publicarlas a través de Flickr, se abre una cuenta y las publica en el servidor, permitiendo que los usuarios puedan verlas, añadir comentarios, reutilizarlas, etc., entonces estaríamos ante un servicio web 2.0.

Imaginemos ahora la biblioteca de un centro universitario. Ha elaborado una selección de sitios web interesantes para sus titulaciones y desea darla a conocer ¿cómo hacerlo? Podría publicarlos en una página web,

\footnotetext{
"El nacimiento del término biblioteca 2.0 y su debate conceptual se han producido en la biblio-blogosfera"
} 
o incluirlas en el opac, o bien optar por el "social bookmarking", creando una cuenta en del.icio.us y compartiéndolas en ese servidor. Otra opción sería instalar un software específico para que sus usuarios puedan crear sus listas de favoritos y compartirlas, como por ejemplo el servicio links de la Yale University Library o, a un nivel mucho más desarrollado, PennTags de la University of Pennsylvania.

\section{Bibliotecas de referencia}

Un ejemplo de lo que pueden ser los servicios biblioteca 2.0 lo constituye la Ann Arbor District Library. Ha convertido su web en un modelo de cómo se puede utilizar la tecnología blog para publicar noticias, novedades bibliográficas o información sobre historia local. Permiten a los usuarios realizar breves comentarios sobre los libros en el catálogo y cuentan con un servicio de fotografías de la ciudad.

Un ejemplo similar en España es la Biblioteca Pública de Muskiz (Bizkaia). Hay que reconocerles varias aportaciones. En primer lugar haber sido pioneros en España en el uso de los blogs para difundir las novedades bibliográficas y crear espacios de participación de los usuarios. Además, han hecho un uso intensivo de las posibilidades que ofrece la web 2.0 y están realizando una importante tarea de difusión de las tecnologías 2.0, no sólo entre sus usuarios, sino también entre las bibliotecas públicas, colaborando con otros centros del Sistema $\mathrm{Na}$ cional de Bibliotecas de Euskadi (Juárez, 2006).

Otra biblioteca que está utilizando servicios 2.0 es la Biblioteca Virtual Miguel de Cervantes. En su catálogo cada obra tiene asociado un foro que permite introducir comentarios y mantener conversaciones escritas sobre ella. Otro caso de innovación en cuanto al catálogo es el de la Lamson Library de la Plymouth State University, que ha realizado el WPopac, basado en WordPress, un programa para publicar blogs. Han implementado APIs que permiten una comunicación en tiempo real entre WordPress, Amazon y el sistema integrado de gestión bibliotecaria de tal forma que WPopac tiene toda la información actualizada, pero presentada de una forma mucho más familiar para los usuarios. Estos dos catálogos serían buenos ejemplos de lo que se denomina "opac social": catálogos de biblioteca que aplican las tecnologías y principios de la web 2.0 en su presentación.

\section{Desarrollo de tecnologías}

El servicio que más se está implantando en las bibliotecas americanas son las "library toolbars", barras de herramientas para los navegadores web donde se integran los servicios esenciales de la biblioteca, entre

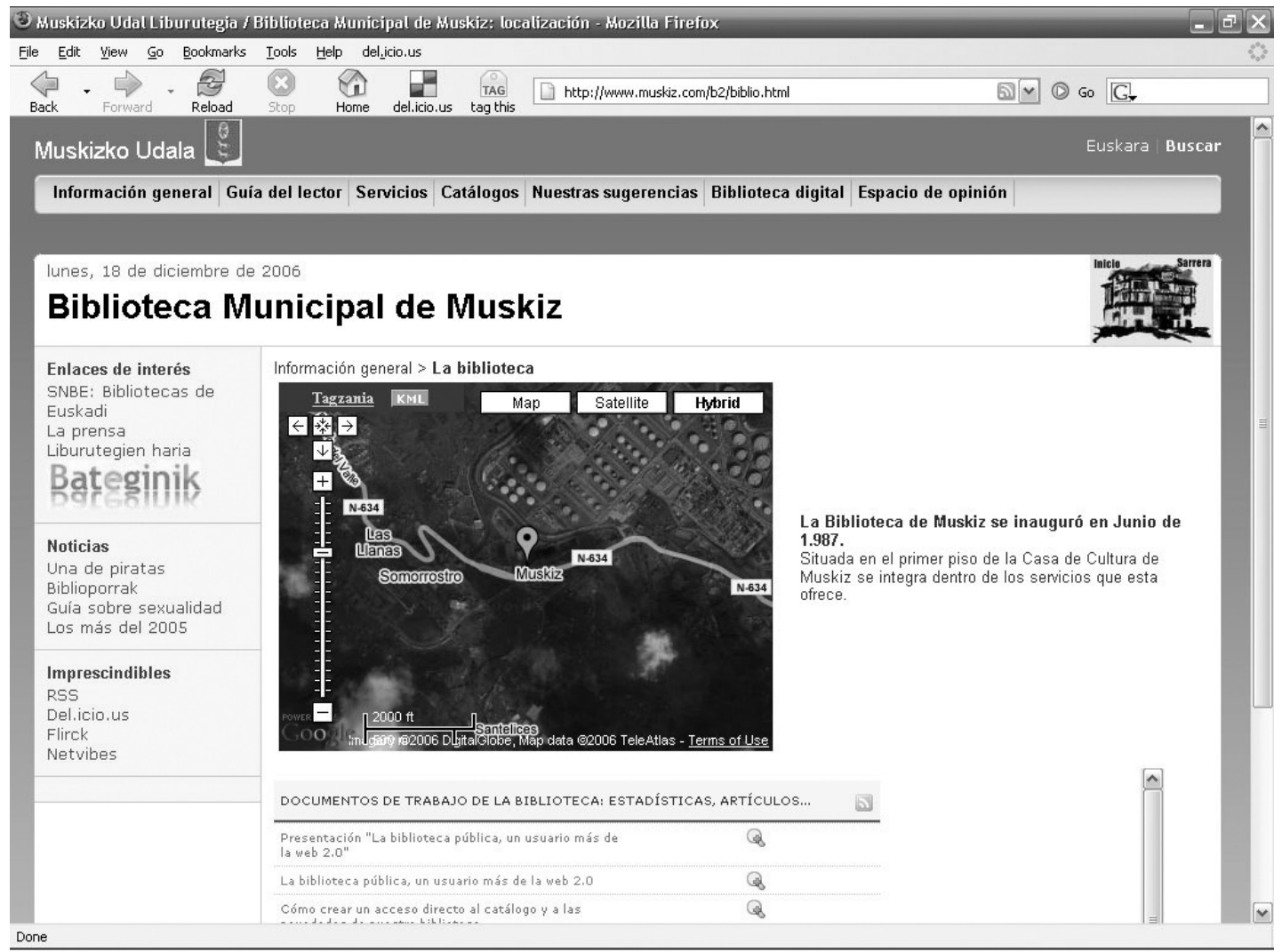

Página principal de la Biblioteca Pública de Muskiz 


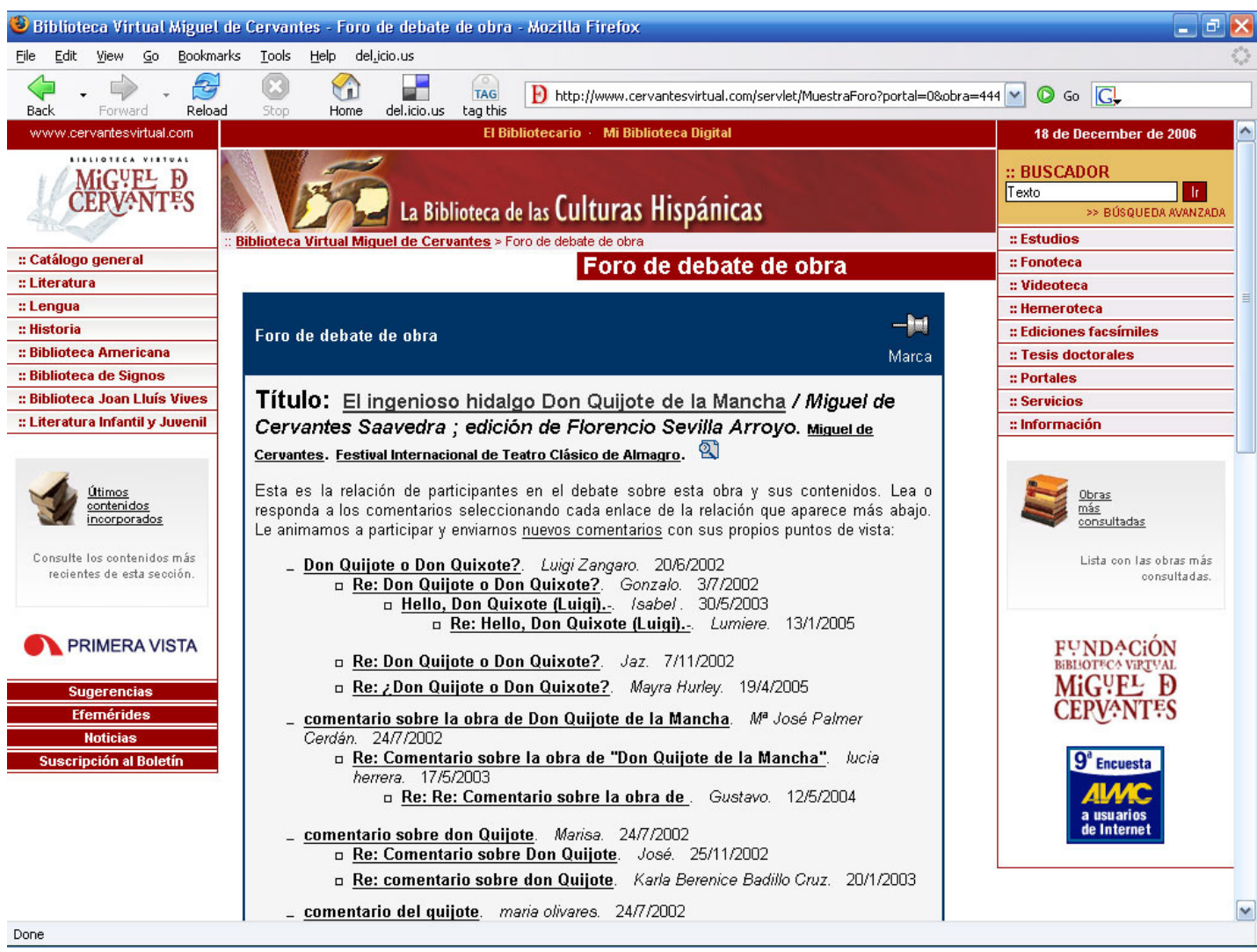

Foro de "El Quijote" en la Biblioteca Virtual Miguel de Cervantes

ellos la búsqueda en el catálogo, referencia, etc. Existe un gran número de ejemplos, pero merece especial atención la University of Pennsylvania. En su barra de herramientas constan todos los servicios esenciales de la universidad, no sólo de la biblioteca. En ella están presentes los más típicos (búsqueda en el catálogo, Google Scholar, acceso al correo electrónico, etc.) pero cuenta con una peculiaridad, un pequeño icono permite añadir la página que se está visitando a PennTags, el "social bookmarking" de la universidad.

Otros servicios 2.0 que pueden llevar a cabo las bibliotecas son: sindicar contenidos de varios canales rss para publicar páginas web que agrupen la información

\section{Infonomia,}

la red de innovadores de referencia

El espacio de referencia para el intercambio de ideas y experiencias sobre la transformación de las organizaciones a través de la innovación.

"Disfruta de las nuevas

secciones, nuevos

protagonistas, nuevas

soluciones"
"Una fuente de ideas

sobre hacia dónde va la empresa en la fusión de personas, procesos, tecnologias y mercados"

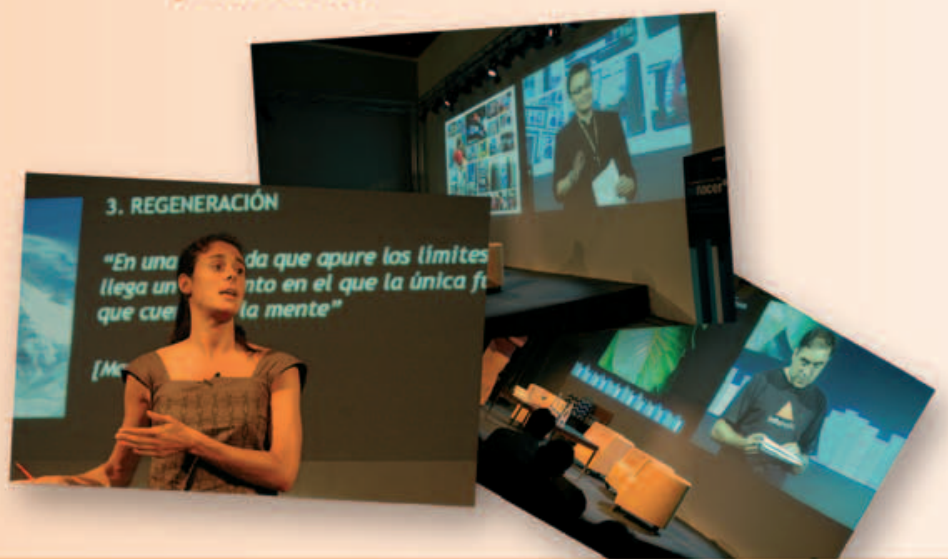




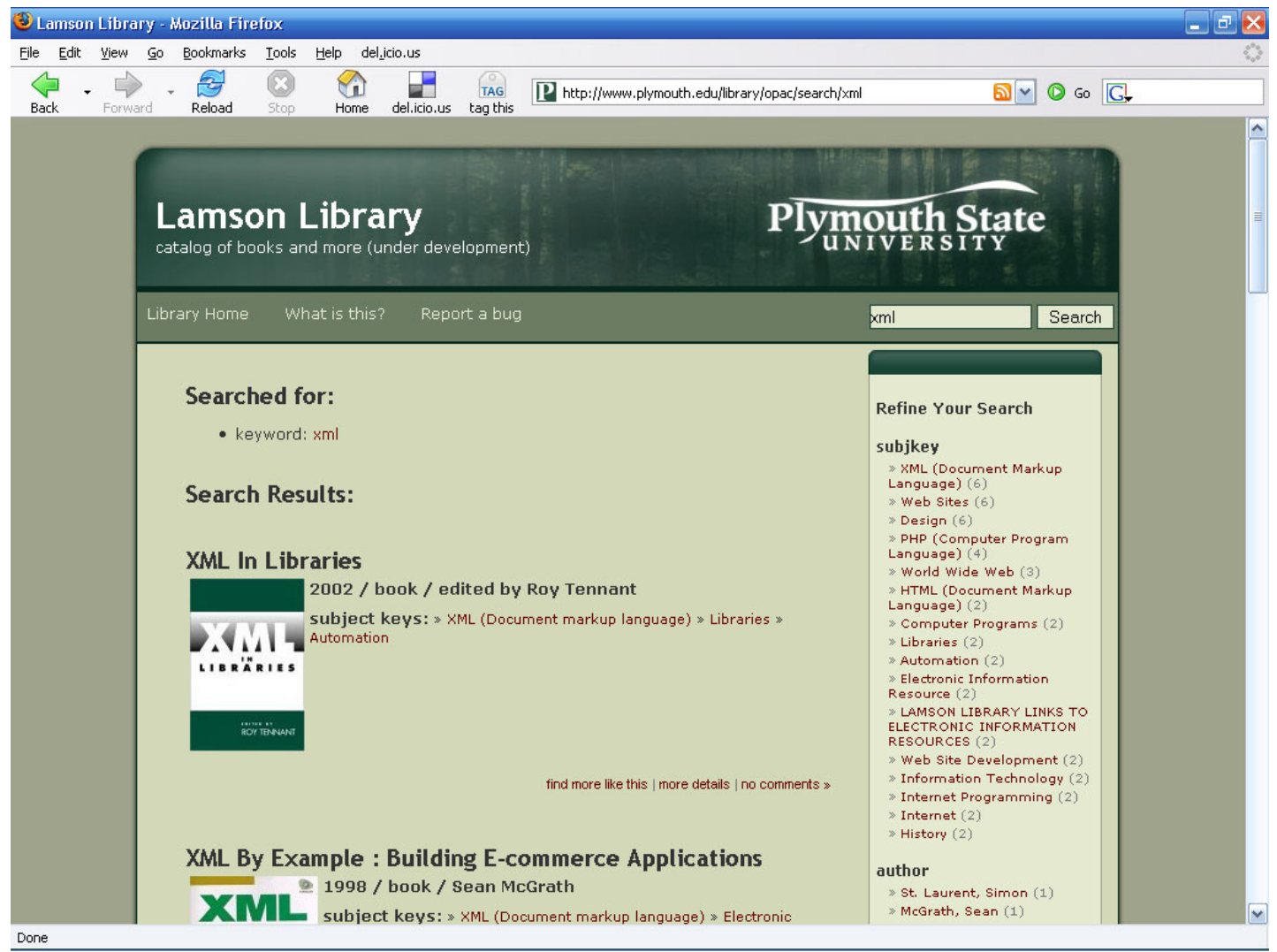

WPopac de la Lamson Library

de interés para los usuarios, crear canales rss de nuevas adquisiciones, desarrollar mashups, realizar alfabetización informacional sobre estas nuevas herramientas, etc. ¿Y los servicios no virtuales? Se podría habilitar un espacio en la biblioteca para el book-crossing entre los usuarios o realizar alfabetización informacional en herramientas de la web 2.0.
"El aprovechamiento de la inteligencia colectiva deberá ser un objetivo para todos los profesionales de la información"

\section{2 próximos números gratuitos...}

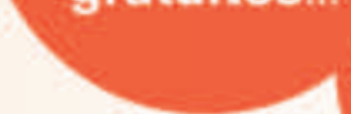

Suscribete a IF...

No dejarás de sorprenderte.

La única revista de innovación del pais

www.infonomia.com/if suscripcion@infonomia.com

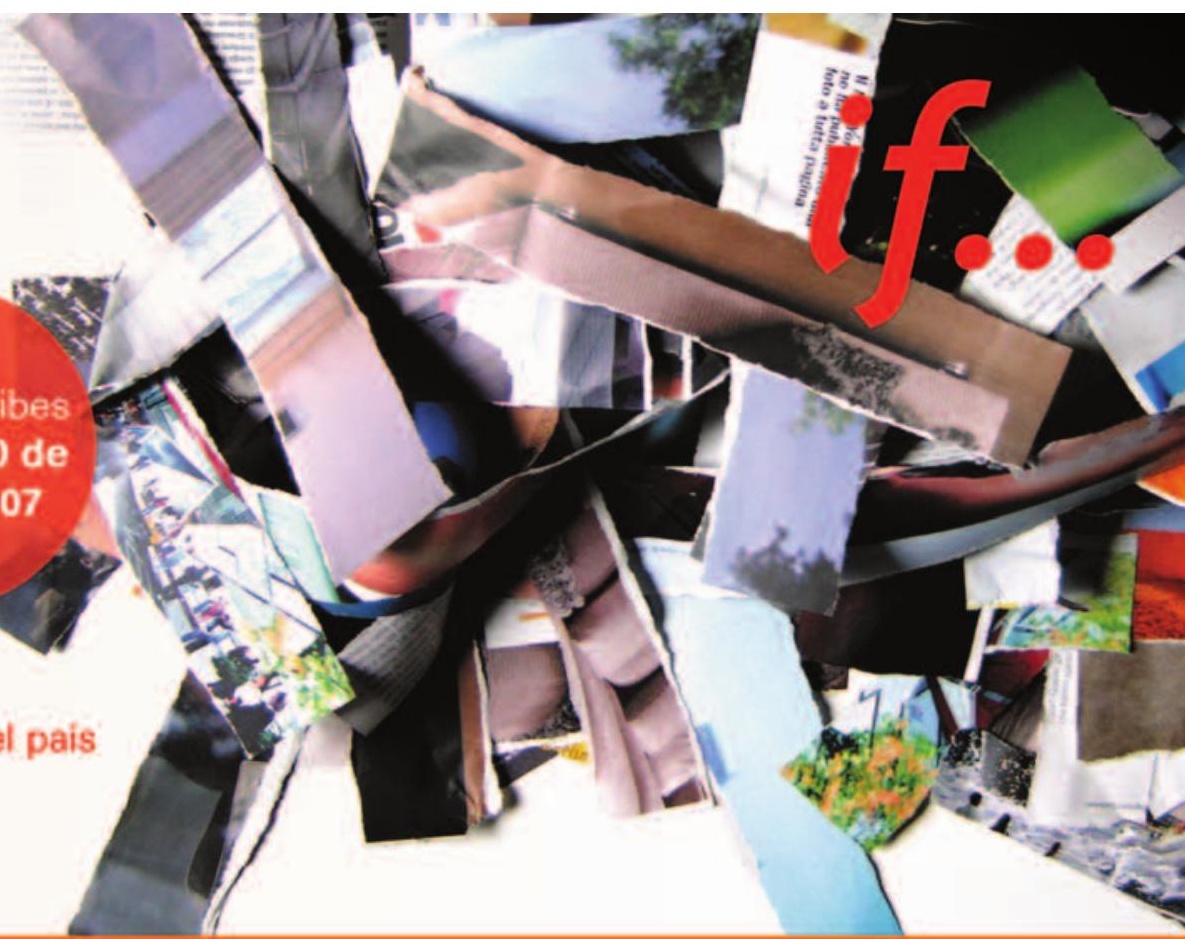




\section{Conclusiones}

El término biblioteca 2.0 hace referencia a la aplicación de determinadas tecnologías y los principios propios de la web 2.0 al entorno bibliotecario. El aprovechamiento de la inteligencia colectiva va a ser un reto importante para todos los profesionales de la información y para afrontarlo es fundamental el conocimiento del software social, pero éste ha de ser sólo una herramienta, no puede convertirse en un objetivo. No se trata de migrar la tecnología o simplemente crear blogs, sino de: usar un nuevo sistema de comunicación con el usuario para que éste enriquezca con su conocimiento nuestros productos informativos; asumir los canales y formas de comunicación actuales y aplicarlos a los servicios bibliotecarios; crear espacios para los lectores, para que interactúen entre ellos y con el personal de la biblioteca, para que puedan opinar, valorar, preguntar, responder, etc.

Es cierto que algunos autores insisten en el hecho de que los servicios de la biblioteca 2.0 no tienen por qué ser sólo electrónicos, que pueden ser también reales o del mundo físico. Si bien es cierto que 2.0 es una actitud, no se puede obviar el hecho de que nace en la industria del software y, por tanto, la mayoría de los servicios que se planteen como 2.0 serán a través de la web.

La existencia de servicios 2.0 es ya un hecho, pero su futuro desarrollo dependerá de las capacidades de los profesionales de la información para adaptarse a las nuevas formas de comunicación, de su capacidad de innovar, de su dominio de las tecnologías 2.0 y de los nuevos productos que ofrezca la industria del software.

\section{Bibliografía}

Austin, Marcus. "The Wikipedia phenomenon". En: Information world review, 2005, Dec., n. 219, pp. 38-39.

Bausch, Paul. Amazon hacks. Sebastopol, CA: O'Reilly, 2003. Isbn 0-59600542-3.

Casey, Michael E.; Savastinuk, Laura C. "Library 2.0”. En: Library journal, 2006, Sept. 1st, v. 131, n. 14, pp. 40-42.

Chad, Ken; Miller, Paul. Do libraries matter?: the rise of library 2.0. Consultado en: 14-11-06.

http://www.talis.com/downloads/white_papers/DoLibrariesMatter.pdf

Clyde, Laurel A. Weblogs and libraries. Oxford: Chandos, 2004. Isbn 184334-085-2.

Crawford, Walt. "Library 2.0 and 'library 2.0"'. En: Cites \& insights, 2006, midwinter, v. 6, n. 2. Consultado en: 14-11-06.

http://cites.boisestate.edu/civ6i2.pdf

Field, Karen Auguston. "Why engineering students [love] Wikipedia". En: Design news, 2006, May, v. 61, n. 7, p. 11.

Franganillo, Jorge; Catalán, Marcos Antonio. "Bitácoras y sindicación de contenidos: dos herramientas para difundir la información". En: BiD: textos universitaris de biblioteconomia i documentació, 2005, diciembre. Consultado en: 18-12-06.

http://www.ub.es/bid/15frang2.htm

Giles, Jim. "Internet encyclopaedias go head to head". En: Nature, 2005, Dec., v. 438, n. 7.070, pp. 900-901.

Gordon-Murnane, Laura. "Social bookmarking, folksonomies and web 2.0 tools". En: Searcher, 2006, June, v. 14, n. 6, pp. 26-38.

Habib, Michael C. (a). Defining academic library 2.0: lessons learned from web 2.0, 5 March 2006. Consultado en: 14-11-06.

http://www.unc.edu/ mchabib/inls342/DefiningAcademicLibrary20Presen tation.pdf

Habib, Michael C. (b). Toward academic library 2.0: development and application of a library 2.0 methodology, 17 Nov. 2006. Consultado en: 18-12-06.

http://hdl.handle.net/1901/356

Juárez Urquijo, Fernando. "La biblioteca pública, un usuario más de la web 2.0". En: III Congreso nacional de bibliotecas públicas, 2006. Consultado en: 18-12-06.

http://www.muskiz.com/b2/Doc/web\%2020\%20y\%20bibliotecas.pdf

Lerner, Reuven M. "At the forge: Google Maps". En: Linux journal, 2006, June, n. 146. Consultado en: 14-11-06.

http://www.linuxjournal.com/article/8932

Maness, Jack M. "Library 2.0 theory: web 2.0 and its implication for libraries”. En: Webology, 2006, June, v. 3, n. 2. Consultado en: 14-11-06. http://www.webology.ir/2006/v3n2/a25.html

Miller, Paul. "Web 2.0: building a new library". En: Ariadne, 2005, Oct., n. 45. Consultado en: 14-11-06.

http://www.ariadne.ac.uk/issue45/miller/

O'Reilly, Tim. (a). What is web 2.0: design patterns and business models for the next generation of software. Publ.: 30 Sept. 2005. Consultado en: 14-11-06.

http://www.oreillynet.com/go/web2

O'Reilly, Tim. (b). Web 2.0: compact definition?, Oct. ${ }^{\text {st }}$, 2005. Consultado en: 14-11-06.

http://radar.oreilly.com/archives/2005/10/web_20_compact_definition.html

Peña, Ismael; Córcoles, César Pablo; Casado, Carlos. "El profesor 2.0: docencia e investigación desde la Red”. En: UOC papers, 2006. n. 3. Consultado en: 18-12-06.

http://www.uoc.edu/uocpapers/3/dt/esp/pena_corcoles_casado.pdf

Rethlefsen, Melissa L. "Product pipeline". En: Netconnect, 2006, summer, pp. 16-17 (suplemento de Library journal).

Ros, Marcos. Un lustro de biblioblogosfera, 9 de noviembre de 2006. Consultado en: 15-11-06.

http://www.documentalistaenredado.net/441/un-lustro-de-biblioblogosfera/

Serrano Cobos, Jorge. Web 2.0 en las bibliotecas: el concepto de library 2.0, 16 de mayo de 2006. Consultado en: 14-11-06.

http://www.thinkepi.net/repositorio/web-20-en-las-bibliotecas-el-conceptolibrary-20/

Sinclair, Mark. "What is Flickr?". En: Creative review, 2006, June, v. 26, n. 6 , pp. 39-41.

Tepper, Michele. "The rise of social software". En: netWorker, 2003, Sept., v. 7, n. 3, pp. 19-23.

Wusteman, Judith. "RSS: the latest feed". En: Library hi tech, 2004, Dec., v. 22, n. 4 , pp. $404-413$.

Dídac Margaix Arnal, Universitat Politècnica de València.

diemarar@bib.upv.es 